\title{
Association of genetic variation in FTO with risk of obesity and type 2 diabetes with data from 96,551 East and South Asians
}

\author{
H. Li • T. O. Kilpeläinen • C. Liu • J. Zhu • Y. Liu • C. Hu • Z. Yang • W. Zhang • \\ W. Bao $\cdot$ S. Cha $\cdot$ Y. Wu $\cdot$ T. Yang $\cdot$ A. Sekine $\cdot$ B. Y. Choi $\cdot$ C. S. Yajnik $\cdot$ D. Zhou

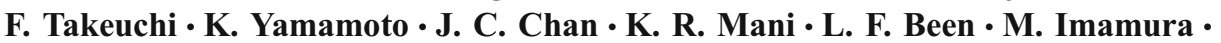 \\ E. Nakashima • N. Lee • T. Fujisawa • S. Karasawa - W. Wen • C. V. Joglekar • W. Lu • \\ Y. Chang • Y. Xiang • Y. Gao • S. Liu • Y. Song • S. H. Kwak • H. D. Shin • K. S. Park • \\ C. H. D. Fall • J. Y. Kim • P. C. Sham • K. S. L. Lam • W. Zheng • X. Shu • H. Deng • \\ H. Ikegami • G. V. Krishnaveni • D. K. Sanghera • L. Chuang • L. Liu • R. Hu • \\ Y. Kim • M. Daimon • K. Hotta • W. Jia • J. S. Kooner • J. C. Chambers • \\ G. R. Chandak $\cdot$ R. C. Ma $\cdot$ S. Maeda $\cdot$ R. Dorajoo $\cdot$ M. Yokota $\cdot$ R. Takayanagi \\ N. Kato $\cdot$ X. Lin $\cdot$ R. J. F. Loos
}

Received: 24 August 2011 / Accepted: 10 October 2011 / Published online: 23 November 2011

(C) The Author(s) 2011. This article is published with open access at Springerlink.com

\begin{abstract}
Aims/hypothesis FTO harbours the strongest known obesity-susceptibility locus in Europeans. While there is growing evidence for a role for FTO in obesity risk in Asians, its association with type 2 diabetes, independently of BMI, remains inconsistent. To test whether there is an association of the FTO locus with obesity and type 2 diabetes, we conducted a meta-analysis of 32 populations including 96,551 East and South Asians.
\end{abstract}

Electronic supplementary material The online version of this article (doi:10.1007/s00125-011-2370-7) contains peer-reviewed but unedited supplementary material, which is available to authorised users.

H. Li $\cdot$ C. Liu $\cdot$ J. Zhu $\cdot$ D. Zhou $\cdot$ X. Lin $(\triangle)$

Institute for Nutritional Sciences,

Shanghai Institutes for Biological Sciences,

Chinese Academy of Sciences,

294 Tai-Yuan Road,

Shanghai 200031, People's Republic of China

e-mail: xlin@sibs.ac.cn

T. O. Kilpeläinen • R. J. F. Loos $(\bowtie)$

MRC Epidemiology Unit, Institute of Metabolic Science Box 285, Addenbrooke's Hospital,

Hills Road,

Cambridge CB2 0QQ, UK

e-mail: ruth.loos@mrc-epid.cam.ac.uk

Y. Liu

Institutes of Biomedical Sciences, Fudan University,

Shanghai, People's Republic of China
Methods All studies published on the association between FTO-rs9939609 (or proxy $\left[r^{2}>0.98\right]$ ) and BMI, obesity or type 2 diabetes in East or South Asians were invited. Each study group analysed their data according to a standardised analysis plan. Association with type 2 diabetes was also adjusted for BMI. Random-effects meta-analyses were performed to pool all effect sizes.

Results The FTO-rs9939609 minor allele increased risk of obesity by 1.25 -fold/allele $\left(p=9.0 \times 10^{-19}\right)$, overweight by

\section{C. $\mathrm{Hu} \cdot$ W. Jia}

Shanghai Diabetes Institute,

Department of Endocrinology and Metabolism,

Shanghai Clinical Center of Diabetes,

Shanghai Jiao Tong University Affiliated Sixth People's Hospital,

Shanghai, People's Republic of China

Z. Yang $\cdot$ R. Hu

Department of Endocrinology and Metabolism, Huashan Hospital, Institute of Endocrinology and Diabetology at Fudan University, Shanghai Medical School, Fudan University,

Shanghai, People's Republic of China

W. Zhang $\cdot$ J. C. Chambers

Department Epidemiology and Biostatistics,

School of Public Health, Imperial College London,

London, UK 
1.13-fold/allele $\left(p=1.0 \times 10^{-11}\right)$ and type 2 diabetes by 1.15 fold/allele $\left(p=5.5 \times 10^{-8}\right)$. The association with type 2 diabetes was attenuated after adjustment for BMI (OR 1.10fold/allele, $p=6.6 \times 10^{-5}$ ). The FTO-rs9939609 minor allele increased BMI by $0.26 \mathrm{~kg} / \mathrm{m}^{2}$ per allele $\left(p=2.8 \times 10^{-17}\right)$, WHR by 0.003 /allele $\left(p=1.2 \times 10^{-6}\right)$, and body fat percentage by $0.31 \% /$ allele $(p=0.0005)$. Associations were similar using dominant models. While the minor allele is less common in East Asians (12-20\%) than South Asians (30$33 \%$ ), the effect of FTO variation on obesity-related traits and type 2 diabetes was similar in the two populations.

Conclusions/interpretation FTO is associated with increased risk of obesity and type 2 diabetes, with effect sizes similar in East and South Asians and similar to those observed in Europeans. Furthermore, FTO is also associated with type 2 diabetes independently of BMI.

\section{W. Bao $\cdot$ L. Liu}

Department of Nutrition and Food Hygiene and MOE Key Lab of Environment and Health, School of Public Health,

Tongji Medical College,

Huazhong University of Science and Technology,

Wuhan, People's Republic of China

\section{S. Cha $\cdot$ J. Y. Kim}

Division of Constitutional Medicine Research,

Korea Institute of Oriental Medicine,

Daejeon, South Korea

Y. $\mathrm{Wu}$

Department of Genetics, University of North Carolina,

Chapel Hill, NC, USA

T. Yang

Key Laboratory of Biomedical Information Engineering of Ministry of Education, and Institute of Molecular Genetics, School of Life Science and Technology, Xi'an Jiaotong University,

Xi'an, People's Republic of China

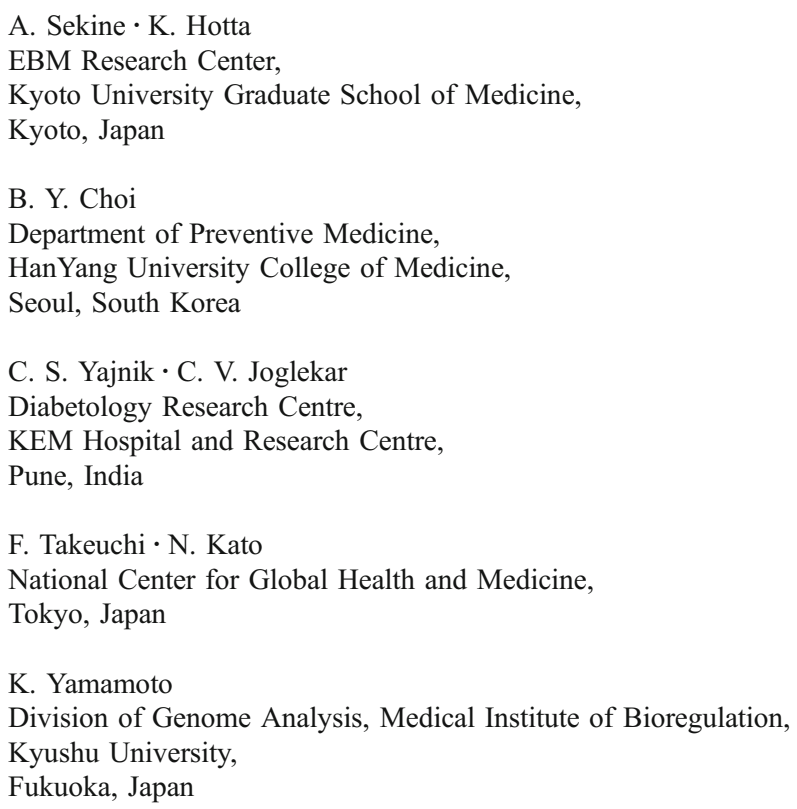

Keywords Asians $\cdot F T O \cdot$ Meta-analysis $\cdot$ Obesity $\cdot$ Type 2 diabetes
Abbreviations
GWAS Genome-wide association study
MAF Minor allele frequency
PAR Population-attributable risk
SNP Single-nucleotide polymorphism

\section{Introduction}

Large-scale genome-wide association studies (GWAS) in mainly white Europeans have identified at least 50 genetic loci to be robustly associated with obesity-related traits [1-

J. C. Chan • R. C. Ma

Department of Medicine and Therapeutics,

Chinese University of Hong Kong, Prince of Wales Hospital,

Shatin, Hong Kong Special Administrative Region,

People's Republic of China

K. R. Mani - G. R. Chandak

Centre for Cellular and Molecular Biology (CCMB-CSIR),

Hyderabad, India

L. F. Been · D. K. Sanghera

University of Oklahoma Health Sciences Center,

Oklahoma City, OK, USA

M. Imamura $\cdot$ S. Maeda

Laboratory for Endocrinology and Metabolism,

RIKEN Center for Genomic Medicine,

Yokohama, Japan

E. Nakashima

Department of Diabetes and Endocrinology,

Chubu Rosai Hospital,

Nagoya, Japan

N. Lee

USC Office of Population Studies Foundation,

University of San Carlos,

Cebu, Philippines

T. Fujisawa

Department of Geriatric Medicine and Nephrology,

Osaka University Graduate School of Medicine,

Suita, Japan

S. Karasawa $\cdot$ M. Daimon

Third Department of Internal Medicine,

and Global Center of Excellence Program Study Group,

Yamagata University School of Medicine,

Yamagata, Japan

W. Wen $\cdot$ W. Zheng $\cdot$ X. Shu

Division of Epidemiology, Department of Medicine,

Vanderbilt School of Medicine,

Nashville, TN, USA 
12]. A cluster of common variants in the first intron of the fat mass and obesity-associated gene (FTO) was the first obesity-susceptibility locus to be identified by two independent GWAS in 2007 [1,2] and has since been consistently replicated by many others and for a variety of obesity-related traits $[7,9,13-15]$. Of all currently identified obesity-susceptibility loci, the FTO locus has the most pronounced effect on BMI and obesity risk, at least in individuals of European descent. Each minor allele of any commonly investigated variant in FTO increases BMI by $0.30-0.40 \mathrm{~kg} / \mathrm{m}^{2}$ (equivalent to $870-1,150 \mathrm{~g}$ for a person $1.7 \mathrm{~m}$ tall) and risk of obesity by $\sim 20 \%[7,15]$. The

\section{W. Lu}

Shanghai Institute of Preventive Medicine,

Shanghai, People's Republic of China

\section{Y. Chang}

National Taiwan University Hospital Bei-Hu branch,

Taipei, Taiwan

\section{Y. Xiang $\cdot$ Y. Gao}

Department of Epidemiology, Shanghai Cancer Institute,

Shanghai, People's Republic of China

S. Liu

Center for Metabolic Disease Prevention, School of Public Health and David Geffen School of Medicine, UCLA,

Los Angeles, CA, USA

\section{Y. Song}

Division of Preventive Medicine, Brigham \& Women's Hospital, Harvard Medical School,

Boston, MA, USA

S. H. Kwak $\cdot$ K. S. Park

Department of Internal Medicine, Seoul National University

College of Medicine,

Seoul, South Korea

H. D. Shin

Department of Life Science, Sogang University,

Seoul, South Korea

C. H. D. Fall

MRC Lifecourse Epidemiology Unit, University of Southampton, Southampton General Hospital,

Southampton, Hampshire, UK

P. C. Sham • K. S. L. Lam

Li Ka Shing Faculty of Medicine, University of Hong Kong,

Hong Kong, Hong Kong Special Administrative Region,

People's Republic of China

\section{H. Deng}

School of Medicine, University of Missouri,

Kansas City, MO, USA

\section{H. Deng}

Center of Systematic Biomedical Research, University of

Shanghai for Science and Technology,

Shanghai, People's Republic of China minor allele of the FTO variant is common (minor allele frequency $(\mathrm{MAF})=\sim 42 \%)$ in white Europeans, such that $66 \%$ of Europeans carry at least one risk allele and $18 \%$ carry two risk alleles. Because of the high prevalence of the risk allele and its relatively strong effect on BMI, the FTO locus explains most $(0.34 \%)$, yet little, of the variation in BMI in Europeans [7].

FTO has also been examined as an obesity-susceptibility locus in populations of non-white European origin. While the initial replication efforts in East Asian populations were inconsistent [16, 17], a growing number of studies have provided evidence that genetic variation in FTO influences

H. Deng

Institute of Bioscience and Biotechnology, School of Science,

Beijing Jiaotong University,

Beijing, People's Republic of China

H. Ikegami

Department of Endocrinology, Metabolism and Diabetes,

Kinki University School of Medicine,

Osaka, Japan

G. V. Krishnaveni

Epidemiology Research Unit, Holdsworth Memorial Hospital, Mysore, India

L. Chuang

Department of Internal Medicine,

National Taiwan University Hospital,

Taipei, Taiwan

Y. Kim

Department of Preventive Medicine,

Dong-A University College of Medicine,

Busan, South Korea

\section{J. S. Kooner}

National Heart \& Lung Institute, Hammersmith Hospital, Hammersmith Campus, Faculty of Medicine,

Imperial College London,

London, UK

R. Dorajoo

Genome Institute of Singapore, Agency for Science,

Technology and Research,

Singapore, Republic of Singapore

R. Dorajoo

Department of Genomics of Common Disease, School of Public Health, Hammersmith Hospital, Imperial College London,

London, UK

M. Yokota

Department of Genome Science, School of Dentistry,

Aichi-Gakuin University,

Nagoya, Japan

R. Takayanagi

Department of Medicine and Bioregulatory Science,

Graduate School of Medical Sciences, Kyushu University,

Fukuoka, Japan 
BMI and obesity risk also in Chinese, Japanese, Korean and Filipino populations [18-27]. A GWAS for BMI in 7,861 Koreans identified variation in FTO (rs9939609) as the most significantly associated locus, nearly reaching genome-wide significance $\left(p=1.5 \times 10^{-7}\right)$ [28]. Furthermore, literature-based meta-analyses in Asians reported that the minor allele for the rs9939609 FTO singlenucleotide polymorphism (SNP) significantly $\left(p=9 \times 10^{-9}\right)$ increased the risk of obesity, but no other obesity-related traits were examined [18, 29, 30]. Fewer studies in South Asians have been reported, two of which confirmed the association between the FTO locus and obesity susceptibility $[31,32]$, whereas one did not [33]. The prevalence of the risk allele in East Asians ( 20\%) and South Asians ( $30 \%)$ is substantially lower than in Europeans, and the reported effect sizes in both East and South Asians vary widely for BMI (OR $0.13-0.83 \mathrm{~kg} / \mathrm{m}^{2}$ per minor allele) and obesity risk (OR 1.02-1.48 per minor allele) [16, 18, 20-25, 27, 34-39].

FTO was first identified as a type 2 diabetessusceptibility gene, but, as further adjustment for BMI abolished the association with type 2 diabetes [1], it was suggested that FTO is primarily an obesity-susceptibility locus. However, the BMI-independent role of FTO in type 2 diabetes remains a matter of debate, particularly in Asians but also in white Europeans. While several studies have reported that the association between the FTO locus and risk of type 2 diabetes remained significant after adjustment for BMI $[15,18,33,35,40,41]$, others could not confirm this $[21,30,32,37,42]$.

To firmly establish the association between the FTO locus and obesity susceptibility in East and South Asians and to assess its effect size and potential heterogeneity across Asian populations, we performed a systematic metaanalysis of data from 32 populations, including 96,551 men and women, using standardised study-specific association analyses. Furthermore, we examined whether the FTO locus is associated with type 2 diabetes independently of its association with BMI.

\section{Methods}

Literature search and study identification We designed a meta-analysis based on de novo analyses of data according to a standardised plan to achieve the greatest consistency possible across studies. We identified all published studies (before September 2010) that had examined the association of genetic variation in FTO with risk of obesity and type 2 diabetes and with obesity-related continuous traits in East and South Asian adults (age $\geq 18$ years) by a PubMed literature search using the key words 'FTO', 'fat mass and obesity associated gene' and 'genome-wide association study'. References from the identified papers were subse- quently screened to identify additional studies and to ensure that the list of eligible studies was complete. The literature search was carried out by two investigators independently, who cross-checked their search results for completeness.

Our literature search identified 38 publications, one of which was excluded because it was a subsample of another identified study. We invited the corresponding authors of the remaining 37 publications to join our meta-analysis, of which 26 agreed to participate and eventually 22 submitted raw data or summary statistics. We also included a Korean population with previously unpublished data (Y. M. Kim, J. Shin, C.B. Lee, M.K. Kim, Y. Tabara, T. Miki and B.Y. Choi), which was presented by a contributing author.

Taken together, our meta-analysis included data for 31 populations from 22 publications and one unpublished study, with 96,551 individuals altogether. The study identification and selection process is illustrated in Fig. 1.

All studies were conducted according to the Declaration of Helsinki. Informed consent was obtained from all participants, and the studies were approved by the ethics committees of the participating institutions.

Genotyping The rs9939609 FTO SNP was examined in 18 studies, whereas proxy SNPs were used in 14 studies. More specifically, the rs8050135 SNP was genotyped in 11 studies of East Asians and one of South Asians, and the rs3751812 and rs17817449 SNP were each genotyped once in studies of East Asians (electronic supplementary material [ESM] Table 1). The linkage disequilibrium between rs9939609 and the three proxies (rs8050135, 3751812, rs 17817449) is perfect $\left(r^{2}=1\right)$ in populations of East Asian origin, based on CHB + JPT data from the HapMap (Rel 24/ Phase II). The linkage disequilibrium between rs9939609 and rs8050135 in Indian Asians is very high $\left(r^{2}>0.98\right)$, based on a subsample $(n=305)$ of the participating Lolipop study.

The genotyping success rate and concordance rate were $>95 \%$, and genotype distributions were in HardyWeinberg equilibrium $(p>0.01)$ in all participating studies (ESM Table 1).

Statistical analysis As case-control definitions and statistical analyses used in the published papers were inconsistent, we asked analysts of each of the participating cohorts to re-analyse their data according to a standardised analysis plan. Summary statistics of each study were subsequently meta-analysed.

Obesity-susceptibility traits and type 2 diabetes Overweight was defined as a BMI $\geq 24 \mathrm{~kg} / \mathrm{m}^{2}$, and obesity as a BMI $\geq 28 \mathrm{~kg} / \mathrm{m}^{2}$ according to the definition proposed by the Working Group on Obesity in China [43]. Anthropometric data, including weight, height, waist circumference, hip 
Fig. 1 Study identification and inclusion in the meta-analyses

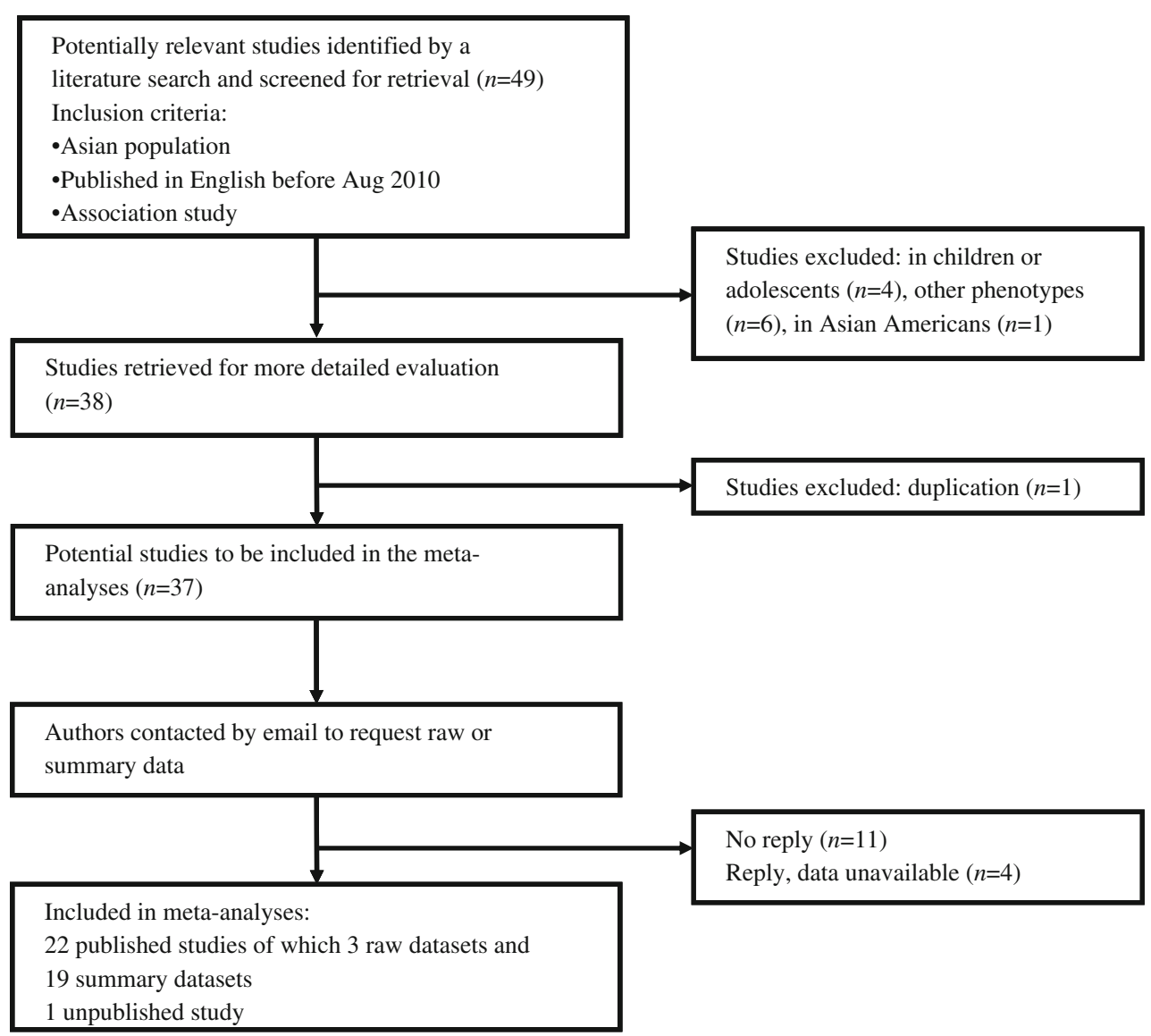

circumference and body fat percentage, were collected in each study as described previously (ESM Table 1), BMI was calculated as weight $(\mathrm{kg})$ divided by height squared $\left(\mathrm{m}^{2}\right)$, and WHR as waist circumference $(\mathrm{cm})$ divided by hip circumference $(\mathrm{cm})$. Raw data were used for analyses.

Type 2 diabetes was defined as meeting one or more of the following criteria: (1) fasting glucose $\geq 7.0 \mathrm{mmol} / \mathrm{l}$; (2) 2 -h glucose $\geq 11.1 \mathrm{mmol} / \mathrm{l}$; (3) previous diagnosis of type 2 diabetes; (4) $\mathrm{HbA}_{1 \mathrm{c}} \geq 6.5 \%$ (48 $\mathrm{mmol} / \mathrm{mol}$ ); (5) selfreported type 2 diabetes (ESM Table 1).

Study-specific de novo data analyses Association analyses within each study were performed for the total population and for men and women separately using additive and dominant genetic models. The associations of FTOrs9939609 (or proxy) with risk of obesity and type 2 diabetes were assessed with multiple logistic regression models. Generalised linear models were used to assess the associations of FTO-rs9939609 (or proxy) with obesityrelated continuous traits. In studies with a case-control design, analyses for continuous traits were conducted in control samples only. All analyses were adjusted for age and sex (sex-stratified analyses were only adjusted for age). The association with type 2 diabetes was also analysed with adjustment for BMI. Adjustments were performed by including the covariates (age, sex and/or BMI) as a linear term in the association model.

Summary statistics from the study-specific association analyses were reported in a standardised Excel form by the analysts of each study and collected centrally for meta-analyses.

Meta-analyses Data extraction from the forms and metaanalyses was performed independently by two investigators and cross-checked for consistency. All ambiguities were clarified with the respective analysts before the final meta-analyses.

ORs and beta coefficients from the individual studies were pooled using DerSimonian and Laird random-effects meta-analyses [44]. Meta-analyses were performed of all studies combined. Because of differences in genetic background as well as in susceptibility to obesity and type 2 diabetes, meta-analyses were also stratified by East Asian and South Asian origin of the populations. Furthermore, East Asians were further stratified according to their country of origin.

Between-study heterogeneity was tested by Cochrane's Q test and quantified by the $I^{2}$ index. $I^{2}$ values of $<25 \%$, $25-75 \%$ and $>75 \%$ were defined as low, moderate and high heterogeneity, respectively [45]. To examine the sources of 
heterogeneity in our meta-analyses, we performed randomeffects meta-regressions, where the between-study variance was estimated with the restricted maximum likelihood approach. Meta-regressions included the following studyspecific variables as covariates: year of publication, country of origin, sample size, study design, mean age and mean BMI.

A funnel plot, along with Begg's and Egger's tests, was used to test for the presence of publication bias.

Statistical analyses were performed with the Stata 9.0 software (StataCorp LP, College Station, TX, USA). Metaanalyses and meta-regressions were implemented by the metan and metareg commands of Stata, respectively. $p<$ 0.05 was considered to be significant, except for Cochrane's Q test for heterogeneity and Begg's and Egger's tests for publication bias, where a level of $p<0.10$ was used.

The variation in obesity-related continuous traits explained by the FTO variant was evaluated using the equation $2 f(1-f) a^{2}$, where $f$ is the frequency of the variant and $a$ is its additive standardised effect [5]. Populationattributable risk (PAR) was calculated as PAR = $(X-1) / X$. Assuming a multiplicative model, $X=$ $(1-f)^{2}+2 f(1-f) \gamma+f^{2} \gamma^{2}$, where $\gamma$ is the estimated $\mathrm{OR}$, and $f$ is the frequency of risk allele [46].

\section{Results}

Characteristics of populations included in the metaanalyses Analyses were conducted in Chinese Hans (China Mainland: $n=10$; Singapore: $n=2)$, Japanese $(n=7)$, Indians $(n=7)$, Koreans $(n=4)$, Singapore Malays $(n=1)$ and Filipinos $(n=1$; Table 1). Fifteen of the populations were case-control designed for obesity $(n=3)$ or type 2 diabetes $(n=8)$ or both $(n=4)$, whereas 17 populations were population-based. The mean age and BMI of the populations ranged from 27.9 to 66.8 years and from 20.5 to $27.1 \mathrm{~kg} / \mathrm{m}^{2}$, respectively. The prevalence in populationbased studies ranged from $3.1 \%$ to $37.9 \%$ for obesity and from $2.9 \%$ to $41.9 \%$ for type 2 diabetes.

The MAF of FTO-rs9939609 (or proxy) is $12-14 \%$ in Chinese Hans and Koreans, $18-20 \%$ in Japanese and Filipinos, and $30-33 \%$ in Singapore Malays and Indians (Table 1).

Associations with obesity and overweight A total of 24 populations $\left(n_{\text {obese }}=13,032 ; n_{\text {overweight }}=22,474 ; n_{\text {normalweight }}=\right.$ $35,767)$ were available for meta-analyses of the association between the FTO variant and risk of obesity and overweight.

Each additional FTO-rs9939609 minor (A) allele increased the odds of obesity by $1.25 \quad\left(p=9.0 \times 10^{-19}\right)$ compared with normal weight individuals (Fig. 2), and by $1.17\left(p=7.4 \times 10^{-11}\right)$ compared with non-obese individuals
(ESM Fig. 1). Each additional minor allele increased the odds of overweight by 1.13 ( $p=1.0 \times 10^{-11}$; ESM Fig. 2$)$. The odds of obesity and overweight were the same in both East Asian and South Asian populations ( $p=0.18$ and 0.84 , respectively; ESM Table 2). Associations were similar in men and women (ESM Table 3). The heterogeneity across all studies was low $\left(13 \% \leq I^{2} \leq 19 \%\right)$.

When a dominant genetic model was used, the odds were only slightly higher than for the additive genetic model (ESM Table 4).

Association with type 2 diabetes In our meta-analysis of 22 populations $\left(n_{\text {cases }}=33,744, n_{\text {controls }}=43,549\right)$, each additional FTO-rs9939609 minor allele increased the odds of type 2 diabetes by $1.15\left(p=5.5 \times 10^{-8}\right)$ when adjusted for age and sex (Fig. 3). Further adjustment for BMI attenuated, but did not abolish, the association with type 2 diabetes (OR 1.10, $p=6.6 \times 10^{-5}$ ) (Fig. 4). Results were similar in East Asians and South Asians (ESM Table 2), in men and women (ESM Table 3), and when a dominant model was used (ESM Table 4).

The association results across all studies showed moderate heterogeneity $\left(44 \% \leq I^{2} \leq 48 \%\right.$; Figs 3 and 4$)$. Meta-regression analyses revealed that the difference in study design contributed to some of the heterogeneity. Subsequent subgroup analyses showed that the association with type 2 diabetes was more pronounced in studies with a case-control design (OR $[95 \% \mathrm{CI}]=1.19[1.14,1.23], p=$ $\left.3.7 \times 10^{-19}, I^{2}=0.0 \%\right)$ than in cohort studies (OR $[95 \% \mathrm{CI}]=$ $1.09[0.99,1.20], p=0.07, I^{2}=54.4 \%$ ), which showed moderate heterogeneity (ESM Table 5).

Associations with obesity-related continuous traits The meta-analyses of the association of FTO-rs9939609 with BMI, waist circumference, hip circumference, WHR and body fat percentage included $30(n=71,022), 22$ $(n=51,543), 20 \quad(n=48,508), 20 \quad(n=48,508)$ and nine $(n=19,580)$ populations, respectively.

Each additional FTO-rs9939609 minor allele was associated with a $0.26 \mathrm{~kg} / \mathrm{m}^{2}$ higher BMI $\left(p=2.8 \times 10^{-17}\right.$; equivalent to $\sim 750 \mathrm{~g} /$ allele for a person $1.7 \mathrm{~m}$ tall) (Fig. 5), $0.51 \mathrm{~cm}$ larger waist circumference $\left(p=3.0 \times 10^{-9}\right)$ (ESM Fig. 3), $0.36 \mathrm{~cm}$ larger hip circumference $(p=0.0003)$ (ESM Fig. 4), 0.003 greater WHR $\left(p=1.2 \times 10^{-6}\right.$; ESM Fig. 5), and $0.31 \%$ higher body fat percentage $(p=0.0005)$ (ESM Fig. 6). All associations were very similar between East and South Asians (ESM Table 2), between men and women (ESM Table 3), or when a dominant genetic model was used (ESM Table 4).

We observed moderate heterogeneity across studies in the associations with BMI and hip circumference (BMI: $I^{2}=33 \%$; hip circumference: $I^{2}=51 \%$; Fig. 5; ESM Fig. 4). Metaregression suggested that, for BMI, the heterogeneity was 


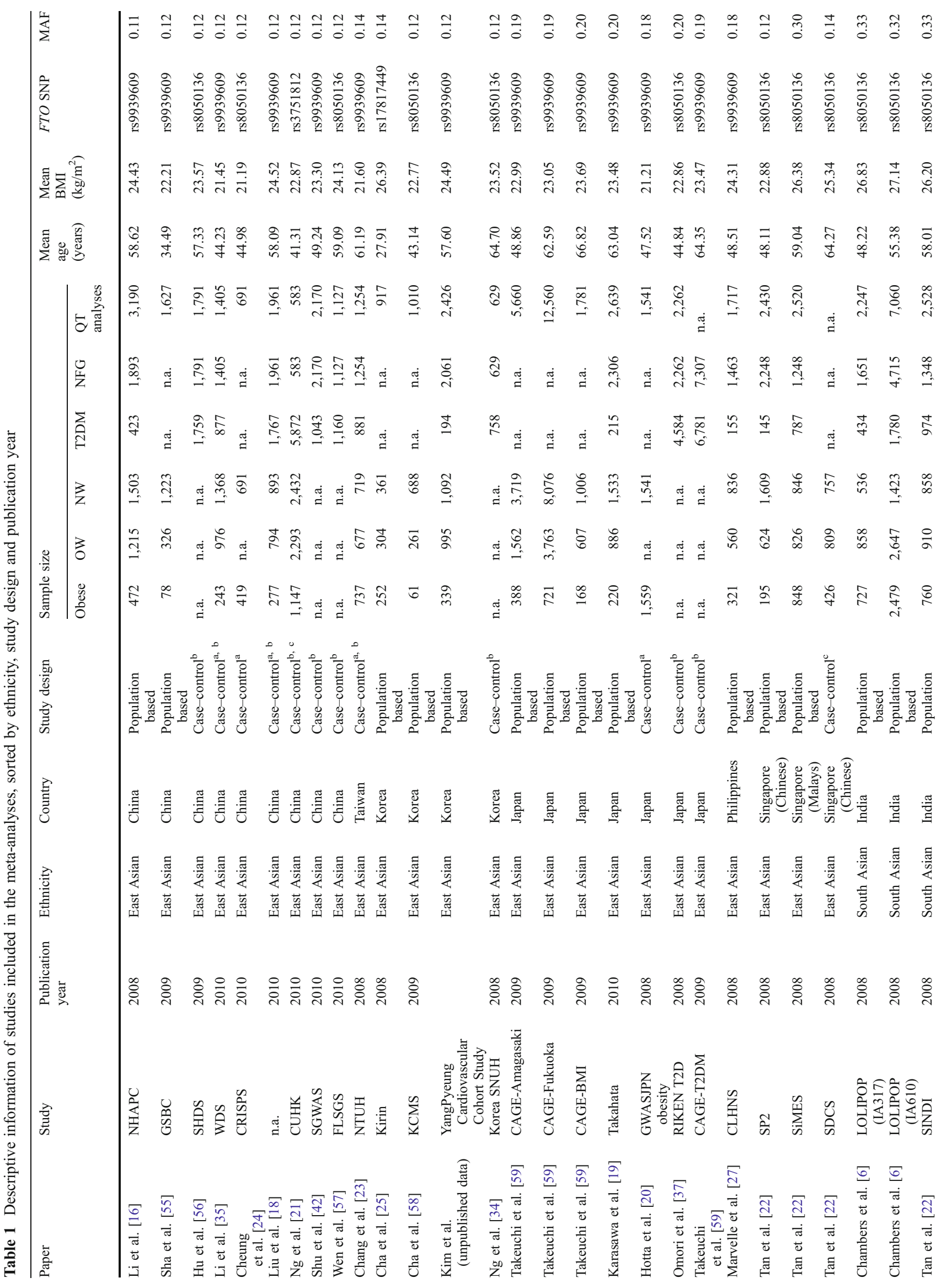




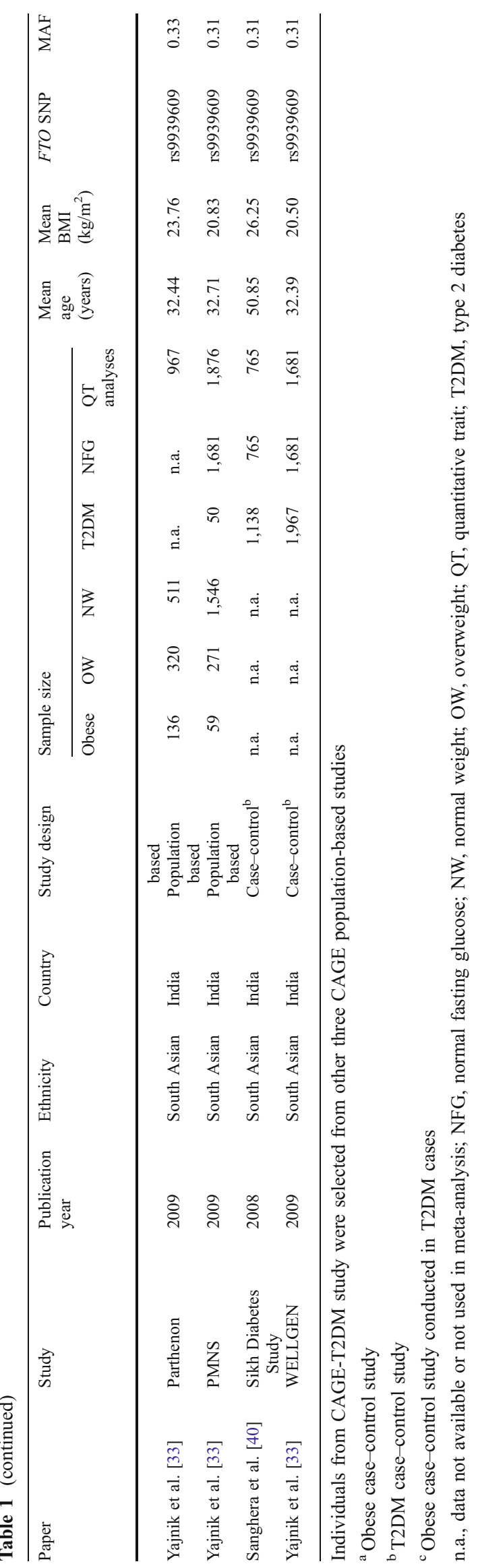

悬 Springer 


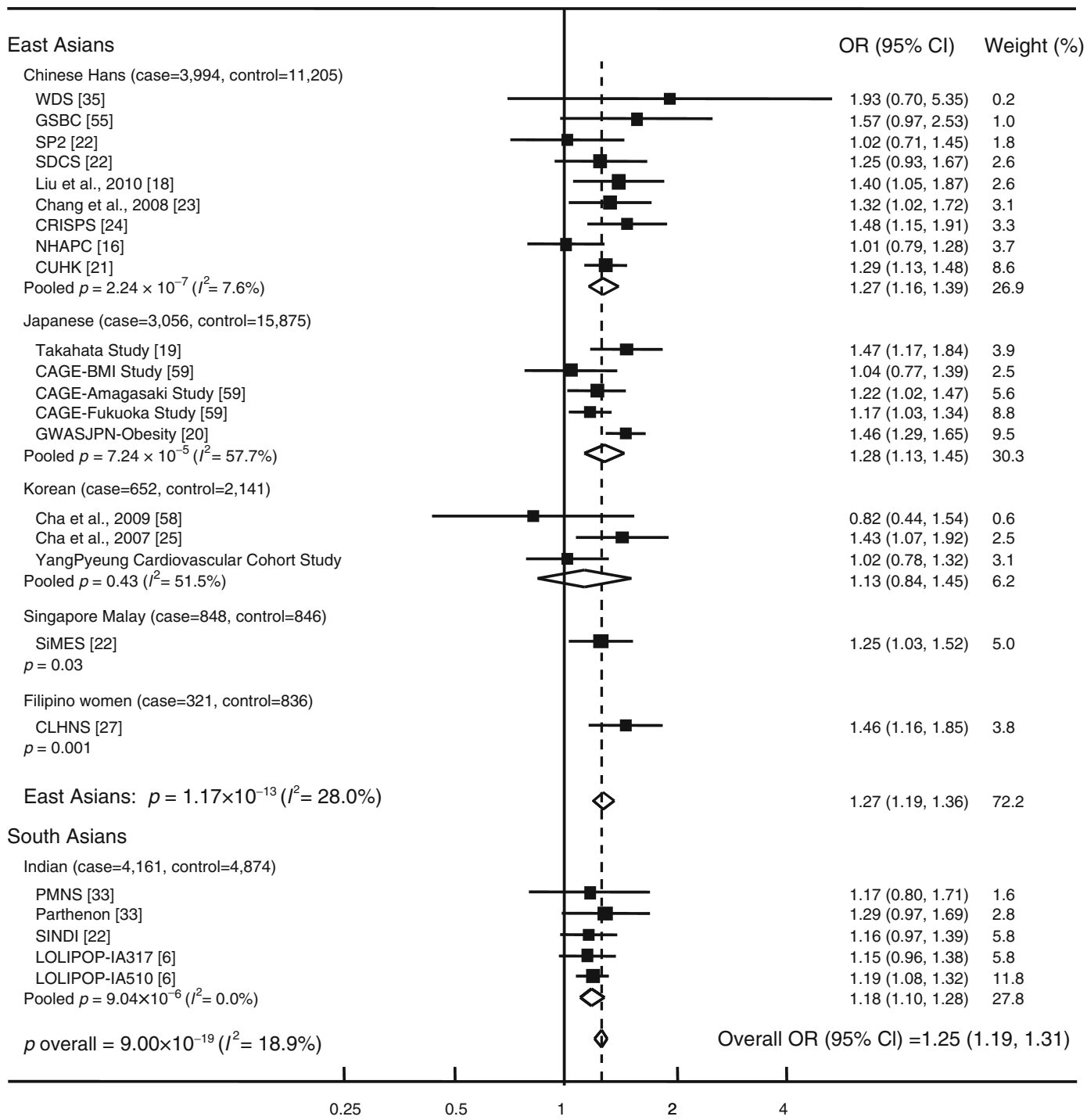

Fig. 2 Association of FTO-rs9939609 (or proxy) with obesity. Studyspecific association analyses assumed an additive genetic model, comparing obese with normal-weight individuals, adjusted for age and

mainly due to difference in mean age and mean BMI among different populations. For hip circumference, the heterogeneity was mainly attributed to difference in mean BMI, i.e. the effect of the FTO minor allele tended to be larger in populations with a mean $\mathrm{BMI} \geq 24 \mathrm{~kg} / \mathrm{m}^{2}$, compared with those with a mean BMI $<24 \mathrm{~kg} / \mathrm{m}^{2}$.

FTO-rs 9939609 explained $0.16 \%$ and $0.20 \%$ of the inter-individual variation in BMI in East and South Asian populations, respectively. The proportion of variation in other obesity-related continuous traits explained by FTO-rs9939609 was $<0.10 \%$ (ESM Table 2).

Publication bias The funnel plots for the associations with obesity, type 2 diabetes, waist circumference, WHR and body fat percentage were symmetrical and the results for Begg's and Egger's tests were non-significant $(p \geq 0.10)$, sex. Effect sizes were combined using random-effects meta-analyses (DerSimonian-Laird method)

indicating that our results were not affected by publication bias (ESM Fig. 7). However, there was some evidence of publication bias and/or genetic heterogeneity for BMI (Begg's test, $p=0.08$; Egger's test, $p=0.07$ ) and hip circumference (Begg's test, $p=0.03$; Egger's test, $p=0.08$; ESM Fig. 7).

\section{Discussion}

This meta-analysis, combining data of 96,551 Asians from 32 populations, further confirms that genetic variation in FTO is associated with increased risk of obesity in East and South Asians. Despite differences in genetic background and obesity susceptibility between East and South Asians, the effect of FTO on obesity and related traits was generally 


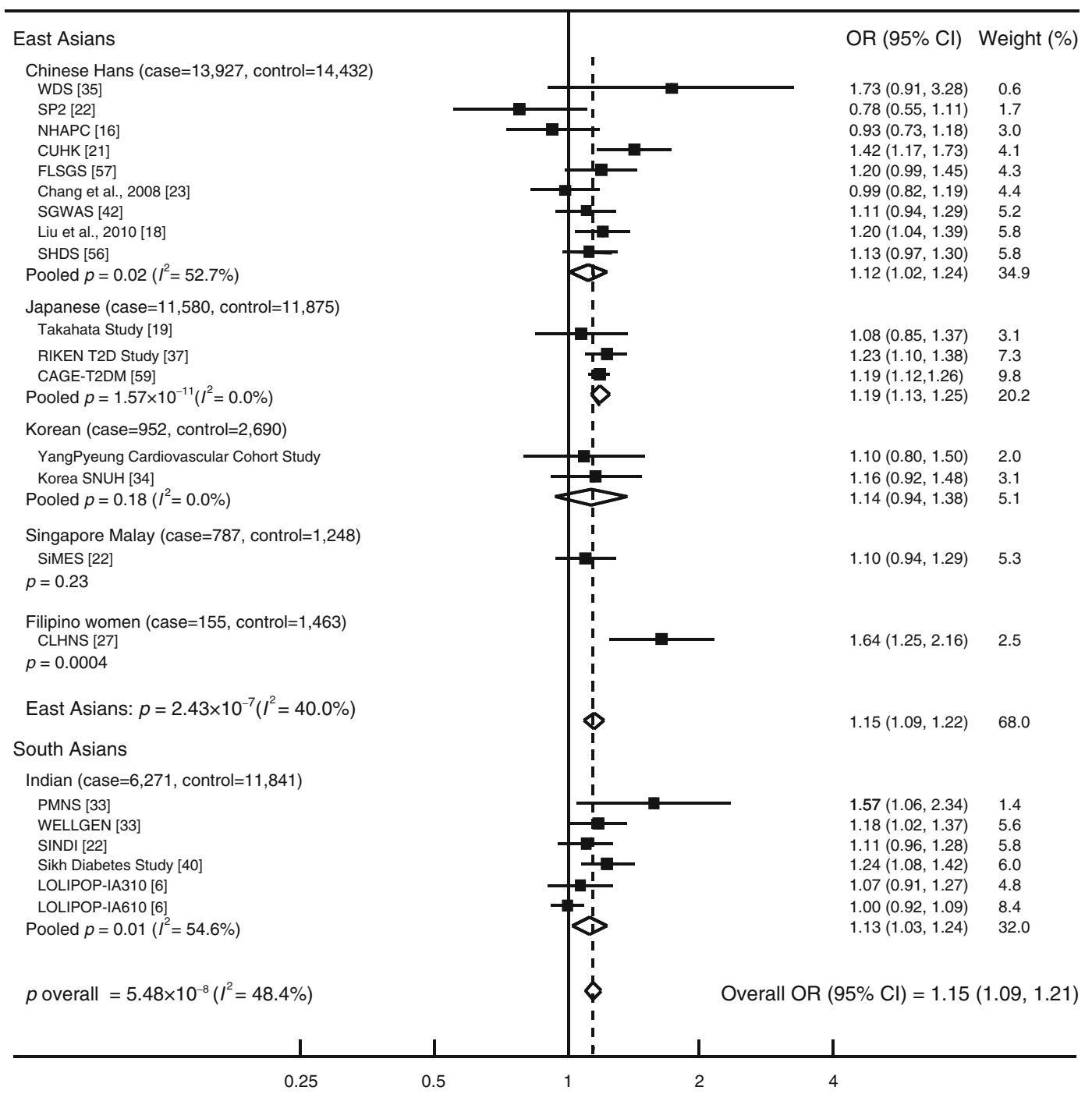

Fig. 3 Association of FTO-rs9939609 (or proxy) with type 2 diabetes. Study-specific association analyses assumed an additive genetic model adjusted for age and sex. Effect sizes were combined using random-effects meta-analyses (DerSimonian-Laird method)

similar to, or only somewhat smaller than, those reported for white Europeans. We furthermore confirm that variation in FTO is associated with increased risk of type 2 diabetes, an association that, unlike in white Europeans, is not abolished after adjustment for BMI in both East and South Asians.

Large-scale studies in individuals of white European descent have reported that each additional FTO minor allele increases the odds of obesity by $1.20-1.32$-fold [1, 5, 7, 47]. The association with obesity observed in Asians in the present study was remarkably similar, with each additional minor allele increasing obesity risk by 1.25 -fold $(95 \%$ CI $1.19,1.31)$, consistent with the association observed for obesity in previous literature-based meta-analyses of casecontrol studies in East and South Asians [18, 29, 30].

The association of the FTO variant with overweight was the same in East and South Asians (OR 1.13 per minor allele) and very similar to the effects (ORs ranging from 1.13 to 1.18 ) that have been reported in large-scale studies of white Europeans [1, 7, 47]. While the effect sizes observed for the influence of FTO on obesity and overweight in Asians are very similar to those of Europeans, it should be noted that the definitions of obesity and overweight are different, as BMI cut-offs are somewhat lower in Asians than in Europeans, consistent with the association of BMI with metabolic disease [48].

The FTO minor allele increases BMI by $0.26 \mathrm{~kg} / \mathrm{m}^{2}$ (equivalent to $\sim 750 \mathrm{~g} /$ allele for a person $1.7 \mathrm{~m}$ tall) in Asians, with very similar results for East and South Asians. This observation suggests that the effect of FTO on BMI in Asians is substantially smaller than the effect observed in a meta-analysis of more than 125,000 white Europeans $\left(0.39 \mathrm{~kg} / \mathrm{m}^{2}\right.$ per minor allele, or $1,130 \mathrm{~g}$ per minor allele $)$ [7]. This difference may be due to the fact that BMI in 


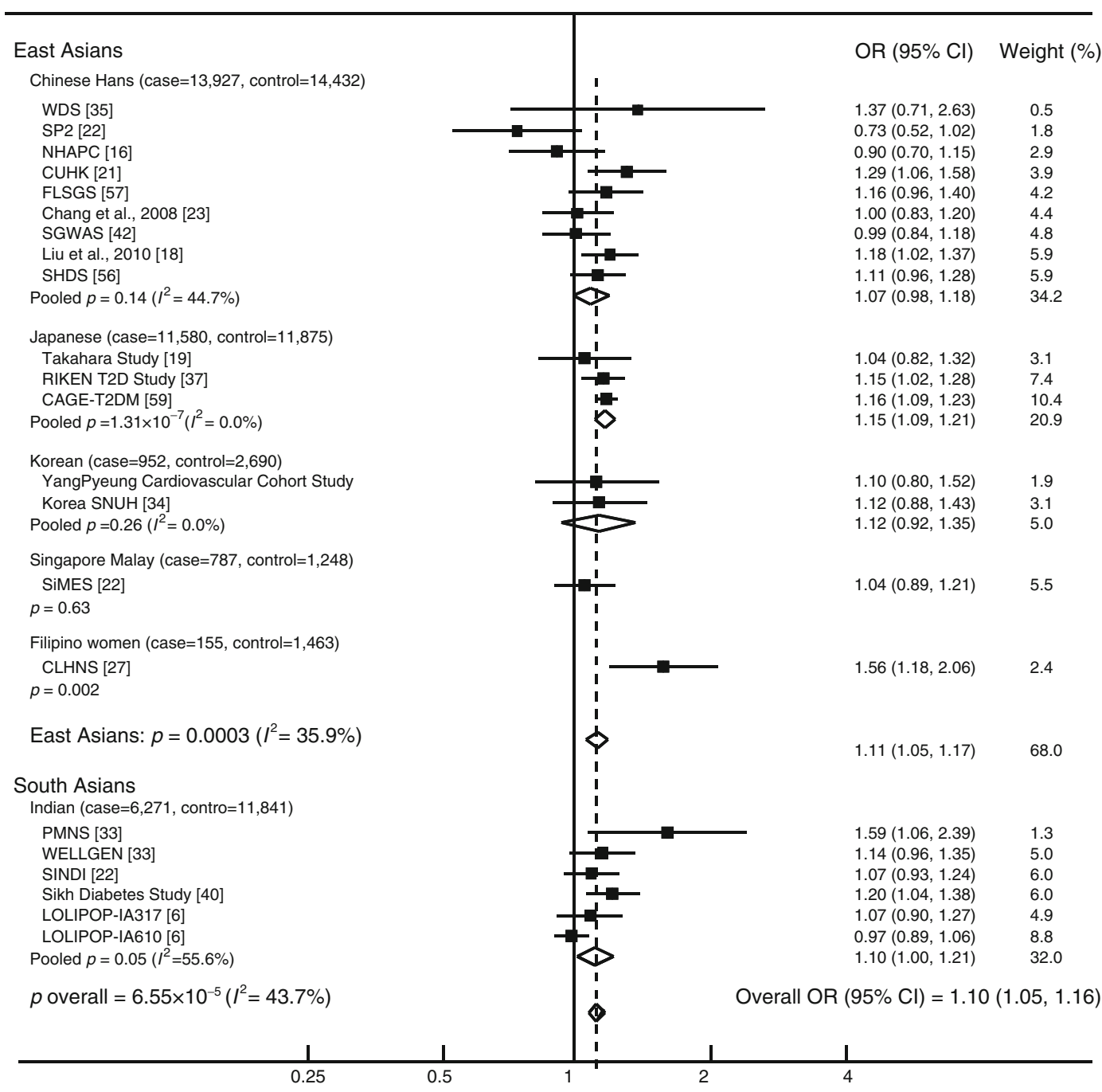

Fig. 4 Association of FTO-rs9939609 (or proxy) with type 2 diabetes adjusted for BMI. Study-specific association analyses assumed an additive genetic model adjusted for age, sex, and BMI. Effect sizes were combined using random-effects meta-analyses (DerSimonian-Laird method)

Asians does not represent exactly the same adiposity phenotype as in Europeans. However, given that other large-scale studies in white Europeans have reported effects for FTO on BMI that range between 0.26 and $0.39 \mathrm{~kg} / \mathrm{m}^{2}$, the comparison between Asians and Europeans should be made with caution $[1,3,5,15,47]$. The $F T O$ variant also showed convincing association with measures of fat distribution such as waist and hip circumference and WHR in Asians. Despite the often described difference in abdominal obesity between East and South Asians, the effect sizes were very similar in the two groups. Consistent with the observations for BMI, the effect sizes tended to be somewhat smaller than those reported for white Europeans. For example, each additional FTO minor allele increased waist circumference by $0.51 \mathrm{~cm}$ in Asians, whereas largescale studies in Europeans have reported an increase of $0.73-1.00 \mathrm{~cm}[1,9,47]$.
As the MAF of the $F T O$ variant is substantially lower in Asians (East Asians, 17\%; South Asians, 32\%) than in white Europeans ( $45 \%)$, and as the effect of this allele on obesity-related traits is similar or somewhat lower in Asians than in white Europeans, the overall contribution of genetic variation in FTO to obesity susceptibility will be lower in Asians, in particular East Asians. For example, the FTO variant explained less of the inter-individual variation in BMI in Asians (East Asians, 0.16\%; South Asians, 0.20\%) than in white Europeans $(0.34 \%)$ [7]. Furthermore, the low risk allele frequency led to a lower PAR for the risk of obesity (East Asians, 8.3\%; South Asians, 10.6\%) and overweight (East Asians, 4.1\%; South Asians, 7.8\%) in Asians than in white Europeans (obesity, 20.4\%; overweight, $12.7 \%$ ) [1].

The FTO locus was first identified in a GWAS for type 2 diabetes in white Europeans, i.e. each minor allele 


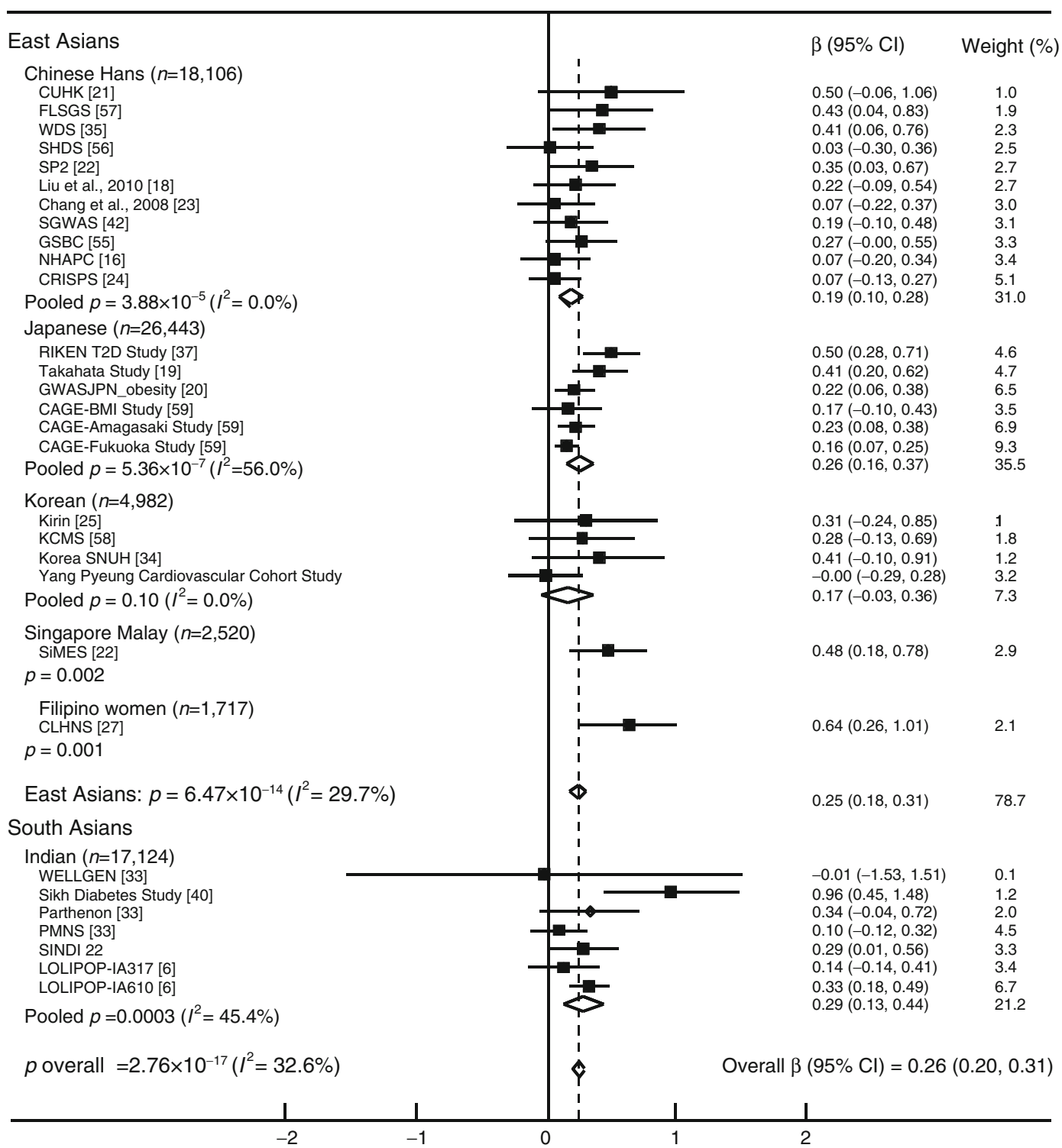

Fig. 5 Association of FTO-rs9939609 (or proxy) with BMI. Study-specific association analyses assumed an additive genetic model adjusted for age and sex. Effect sizes were combined using random-effect meta-analyses (DerSimonian-Laird method)

increased the odds of diabetes by 1.15-fold [1]. However, after adjustment for BMI, the association between the FTO variant and type 2 diabetes was completely abolished (OR 1.03 ), suggesting that FTO is primarily an obesitysusceptibility locus [1]. In our meta-analysis, we observed a similar effect of FTO on risk of type 2 diabetes, with each minor allele increasing the odds by 1.15 -fold. Interestingly, adjustment for BMI did not abolish the association, but only slightly attenuated it to a 1.10 -fold increased risk of type 2 diabetes for each additional minor allele. These observations were similar in East and South Asians, suggesting that the FTO locus influences the risk of type 2 diabetes, at least in part, independently of its effect on BMI. The reason for the discrepancy between the original observations in Europeans and our observations in Asians are not known, but may be due to the fact that FTO seems to have a smaller effect on BMI in Asians than in Europeans. It may also be due to the fact that BMI, as suggested above, represents a different adiposity phenotype in Asians than in Europeans because of differences in body composition. Although BMI is a marker for general adiposity, it does not distinguish between fat mass and fatfree mass and does not reflect regional fat distribution. Observational studies have suggested that, for a given amount of total body fat, East and South Asians have more abdominal fat and less muscle mass than white Europeans $[49,50]$. However, while it has been generally believed that in white Europeans the association with type 2 
diabetes is fully mediated by the effect of FTO on BMI, not all studies confirm this observation. A recent largescale study in 41,504 Scandinavians found that the FTO minor allele indeed increased type 2 diabetes risk (OR 1.13), but this association remained present (OR 1.09) after adjustment for BMI, consistent with the observations in the present study. The biological pathways that underlie the independent association between FTO variation with obesity and type 2 diabetes remain unclear. However, results of gene expression studies have shown that FTO expression in human islets cells is not associated with BMI [51], whereas FTO mRNA and protein levels in muscle are increased in individuals with type 2 diabetes compared with non-diabetic obese individuals or healthy lean controls [52]. Furthermore, FTO overproduction in myotubes suggested a role for FTO in oxidative metabolism, lipogenesis and oxidative stress in muscle, a cluster of metabolic defects characteristic of type 2 diabetes [52].

Despite the fact that our meta-analyses included Asians with different genetic backgrounds, the overall heterogeneity of the association effects was generally only low to moderate. Interestingly, we found that the associations were generally very similar in East and South Asians, although these populations are known to have genetically different origins [53]. Furthermore, we found no differences between men and women, consistent with the observations in white Europeans [7]. We found some evidence that age may contribute to the heterogeneity of the association between FTO and BMI. Life course effects have been reported in white Europeans [54], and longitudinal analyses will be needed to establish this in Asian populations. Longitudinal studies are also more appropriate than cross-sectional studies for disentangling the intricate interplay between FTO, obesity and type 2 diabetes throughout life [15].

It should be noted that the association between FTO variation and obesity risk in Asians had been established in three earlier meta-analyses $[18,29,30]$. These metaanalyses were substantially smaller than the present ones and focused solely on case-control analyses of obesity and type 2 diabetes, while no continuous traits were studied. The meta-analysis by Liu et al [18] included individuals of East and South Asian origin, which were analysed together without comparison of effect sizes between the two populations. This study also examined the association with type 2 diabetes, but did not explore the association after adjustment for BMI [18]. Furthermore, the three previous meta-analyses were all literature-based and thus more prone to publication bias, whereas our meta-analysis was designed on the basis of a de novo analysis of data according to a standardised plan in all studies identified as having available data and agreement to participate. No evidence of publication bias was observed except for the associations with BMI and hip circumference. The analytical consistency across studies helped minimise betweenstudy heterogeneity. Although our results are representative of individuals of Southeast Asian, East Asian and South Asian descent, the association of FTO with risk of obesity and type 2 diabetes in other Asian populations remains to be examined.

In summary, we have firmly established that genetic variation in the first intron of FTO is associated with increased risk of obesity and type 2 diabetes in Asians, with effect sizes similar to those in Europeans. Furthermore, we confirm that the association of FTO with risk of type 2 diabetes is partly independent of BMI.

Acknowledgements A full list of acknowledgements can be found in the ESM. This work was supported by the following: Hong Kong Government Research Grants Council Central Allocation Scheme (CUHK 1/04C), Research Grants Council Earmarked Research Grant (CUHK4724/07M) and the Innovation and Technology Fund of the Government of the Hong Kong SAR (ITS/487/09FP); National Institutes of Health (DK078150, TW05596, HL085144, TW008288) and pilot funds (RR20649, ES10126, and DK56350); National Institutes of Health (R01 AR050496, R21 AG027110, R01 AG026564, R01 AR057049-01A1, R21 AA015973) and Specialized Center of Research (P50 AR055081) and National Science Foundation of China $(81000363,31000554)$ and the PhD Programs Foundation of Ministry of Education of China (20100201120058) and the Fundamental Research Funds for the Central Universities, Shanghai Leading Academic Discipline Project (S30501); National Research Foundation of Korea (NRF, no. 20100020617); the Japanese Society for the Promotion of Science (17209021) and of Priority Area 'Applied Genomics' (1601223, 17019028, 18018020, 20018026); the Korea Health 21 R\&D Project, Korean Ministry of Health \& Welfare (00-PJ3-PG6-GN07-001); the National Institute for Health Research Comprehensive Biomedical Research Centre at Imperial College Healthcare NHS Trust, the British Heart Foundation (SP/04/002), the Medical Research Council (G0700931), the Wellcome Trust (084723/ $\mathrm{Z} / 08 / \mathrm{Z}$ ) and the National Institute for Health Research (RP-PG-040710371); the Knowledge Innovation Program of Shanghai Institutes for Biological Sciences, Chinese Academy of Sciences (2009KIP401), the Chief Scientist Program of Shanghai Institutes for Biological Sciences, Chinese Academy of Sciences (SIBS2008006), the Knowledge Innovation Program Project of the Chinese Academy of Sciences (KSCX1-YW-02), the National Natural Science Foundation of China (30930081), and National High Technology Research and Development Program (863 Program) (2009AA022704), National 973 Program (2011CB504002); the National Science Council (NSC 95-3112-B002-002, NSC 96-2752-B002-008-PAE) of Taiwan; major program of Shanghai Municipality for Basic Research (08dj1400601), National Natural Science Foundation of China (30800617); National Institute of Health (NIH/FIC KO1 TW006087, NIH/NIDDK R01 DK082766); the Program for the National Natural Science Foundation of China (NSFC-30872116) and New Century Excellent Talents in the University of China (NCET-04-0707); Council of Scientific and Industrial Research, Government of India (NWP0032); the National Heart, Lung, and Blood Institute, National Institutes of Health, US Department of Health and Human Services (N01WH22110, 24152, 32100-2, 32105-6, 32108-9, 32111-13, 32115, 32118-32119, 32122, 42107-26, 42129-32, 44221) and the National Institute of Diabetes and Digestive and Kidney Diseases R01 (DK062290) from the National Institutes of Health. 
Contribution statement RJFL, HL and XL contributed to the conception and design of the study. CL and TOK performed the literature search, designed the analysis plan, performed the metaanalyses and researched the data. HL, TOK, CL and RJFL wrote the manuscript. HL, JZ, YL, CH, ZY, WZ, WB, SC, YW, TY, AS, BYC, CSY, DZ, FT, KY, JCC, KRM, LFB, MI, EN, NL, TF, SK, WW, CVJ, WL, YC, YX, YG, SL, YS, SHK, HDS, KSP, CHDF, JYK, PCS, KSLL, WZ, XS, HD, HI, GVK, DKS, LC, LL, RH, YK, MD, KH, WJ, JSK, JCC, GRC, RCM, SM, RD, MY, RT, NK, XL and RJFL collected study-specific data, analysed the study-specific data according to the standardised analysis plan, and reviewed and edited the manuscript. All authors have approved the final version of the manuscript to be published.

Duality of interest The authors declare that there is no duality of interest associated with this manuscript.

Open Access This article is distributed under the terms of the Creative Commons Attribution Noncommercial License which permits any noncommercial use, distribution, and reproduction in any medium, provided the original author(s) and source are credited.

\section{References}

1. Frayling TM, Timpson NJ, Weedon MN et al (2007) A common variant in the FTO gene is associated with body mass index and predisposes to childhood and adult obesity. Science 316:889-894

2. Scuteri A, Sanna S, Chen WM et al (2007) Genome-wide association scan shows genetic variants in the FTO gene are associated with obesity-related traits. PLoS Genet 3:e115

3. Loos RJ, Lindgren CM, Li S et al (2008) Common variants near MC4R are associated with fat mass, weight and risk of obesity. Nat Genet 40:768-775

4. Willer CJ, Speliotes EK, Loos RJ et al (2009) Six new loci associated with body mass index highlight a neuronal influence on body weight regulation. Nat Genet 41:25-34

5. Thorleifsson G, Walters GB, Gudbjartsson DF et al (2009) Genome-wide association yields new sequence variants at seven loci that associate with measures of obesity. Nat Genet 41:18-24

6. Chambers JC, Elliott P, Zabaneh D et al (2008) Common genetic variation near MC4R is associated with waist circumference and insulin resistance. Nat Genet 40:716-718

7. Speliotes EK, Willer CJ, Berndt SI et al (2010) Association analyses of 249,796 individuals reveal 18 new loci associated with body mass index. Nat Genet 42:937-948

8. Lindgren CM, Heid IM, Randall JC et al (2009) Genome-wide association scan meta-analysis identifies three loci influencing adiposity and fat distribution. PLoS Genet 5:e1000508

9. Heard-Costa NL, Zillikens MC, Monda KL et al (2009) NRXN3 is a novel locus for waist circumference: a genome-wide association study from the CHARGE Consortium. PLoS Genet 5:e1000539

10. Heid IM, Jackson AU, Randall JC et al (2010) Meta-analysis identifies 13 new loci associated with waist-hip ratio and reveals sexual dimorphism in the genetic basis of fat distribution. Nat Genet 42:949-960

11. Meyre D, Delplanque J, Chevre JC et al (2009) Genome-wide association study for early-onset and morbid adult obesity identifies three new risk loci in European populations. Nat Genet $41: 157-159$

12. Scherag A, Dina C, Hinney A et al (2010) Two new Loci for body-weight regulation identified in a joint analysis of genome- wide association studies for early-onset extreme obesity in French and German study groups. PLoS Genet 6:e1000916

13. Kring SI, Holst C, Zimmermann E et al (2008) FTO gene associated fatness in relation to body fat distribution and metabolic traits throughout a broad range of fatness. PLoS One 3:e2958

14. Hotta K, Nakamura M, Nakamura $T$ et al (2010) Polymorphisms in NRXN3, TFAP2B, MSRA, LYPLAL1, FTO and MC4R and their effect on visceral fat area in the Japanese population. J Hum Genet 55:738-742

15. Hertel JK, Johansson S, Sonestedt E et al (2011) FTO, Type 2 diabetes, and weight gain throughout adult life: a meta-analysis of 41,504 subjects from the Scandinavian HUNT, MDC, and MPP studies. Diabetes 60:1637-1644

16. Li H, Wu Y, Loos RJ et al (2008) Variants in the fat mass- and obesity-associated (FTO) gene are not associated with obesity in a Chinese Han population. Diabetes 57:264-268

17. Horikoshi M, Hara K, Ito C et al (2007) Variations in the HHEX gene are associated with increased risk of type 2 diabetes in the Japanese population. Diabetologia 50:2461-2466

18. Liu Y, Liu Z, Song Y et al (2010) Meta-analysis added power to identify variants in FTO associated with type 2 diabetes and obesity in the Asian population. Obesity (Silver Spring) 18:1619 1624

19. Karasawa S, Daimon M, Sasaki S et al (2010) Association of the common fat mass and obesity associated (FTO) gene polymorphism with obesity in a Japanese population. Endocr J 57:293301

20. Hotta K, Nakata Y, Matsuo T et al (2008) Variations in the FTO gene are associated with severe obesity in the Japanese. J Hum Genet 53:546-553

21. $\mathrm{Ng} \mathrm{MC}$, Tam CH, So WY et al (2010) Implication of genetic variants near NEGR1, SEC16B, TMEM18, ETV5/DGKG, GNPDA2, LIN7C/BDNF, MTCH2, BCDIN3D/FAIM2, SH2B1, $F T O, M C 4 R$, and $K C T D 15$ with obesity and type 2 diabetes in 7705 Chinese. J Clin Endocrinol Metab 95:2418-2425

22. Tan JT, Dorajoo R, Seielstad M et al (2008) FTO variants are associated with obesity in the Chinese and Malay populations in Singapore. Diabetes 57:2851-2857

23. Chang YC, Liu PH, Lee WJ et al (2008) Common variation in the fat mass and obesity-associated (FTO) gene confers risk of obesity and modulates BMI in the Chinese population. Diabetes 57:22452252

24. Cheung CY, Tso AW, Cheung BM et al (2010) Obesity susceptibility genetic variants identified from recent genome-wide association studies: implications in a Chinese population. J Clin Endocrinol Metab 95:1395-1403

25. Cha SW, Choi SM, Kim KS et al (2008) Replication of genetic effects of FTO polymorphisms on BMI in a Korean population. Obesity (Silver Spring) 16:2187-2189

26. Lee HJ, Kim IK, Kang JH et al (2010) Effects of common FTO gene variants associated with BMI on dietary intake and physical activity in Koreans. Clin Chim Acta 411:1716-1722

27. Marvelle AF, Lange LA, Qin L, Adair LS, Mohlke KL (2008) Association of FTO with obesity-related traits in the Cebu Longitudinal Health and Nutrition Survey (CLHNS) Cohort. Diabetes 57:1987-1991

28. Cho YS, Go MJ, Kim YJ et al (2009) A large-scale genome-wide association study of Asian populations uncovers genetic factors influencing eight quantitative traits. Nat Genet 41:527-534

29. Peng S, Zhu Y, Xu F, Ren X, Li X, Lai M (2011) FTO gene polymorphisms and obesity risk: a meta-analysis. BMC Med 9:71

30. Xi B, Mi J (2009) FTO polymorphisms are associated with obesity but not with diabetes in East Asian populations: a metaanalysis. Biomed Environ Sci 22:449-457 
31. Dorajoo R, Blakemore AI, Sim X et al (2011) Replication of 13 obesity loci among Singaporean Chinese. Malay and AsianIndian populations. Int J Obes (Lond). doi:10.1038/ijo.2011.86

32. Ramya K, Radha V, Ghosh S, Majumder PP, Mohan V (2010) Genetic variations in the FTO gene are associated with type 2 diabetes and obesity in south Indians (CURES-79). Diabetes Technol Ther 13:33-42

33. Yajnik CS, Janipalli CS, Bhaskar S et al (2009) FTO gene variants are strongly associated with type 2 diabetes in South Asian Indians. Diabetologia 52:247-252

34. Ng MC, Park KS, Oh B et al (2008) Implication of genetic variants near TCF7L2, SLC30A8, HHEX, CDKAL1, CDKN2A/B, $I G F 2 B P 2$, and FTO in type 2 diabetes and obesity in 6,719 Asians. Diabetes 57:2226-2233

35. Li X, Song F, Jiang $\mathrm{H}$ et al (2010) A genetic variation in the fat mass- and obesity-associated gene is associated with obesity and newly diagnosed type 2 diabetes in a Chinese population. Diabetes Metab Res Rev 26:128-132

36. Shi J, Long J, Gao YT et al (2010) Evaluation of genetic susceptibility loci for obesity in Chinese women. Am J Epidemiol 172:244-254

37. Omori S, Tanaka Y, Takahashi A et al (2008) Association of $C D K A L 1, I G F 2 B P 2, C D K N 2 A / B$, HHEX, SLC30A8, and KCNJ11 with susceptibility to type 2 diabetes in a Japanese population. Diabetes 57:791-795

38. Tabara Y, Osawa H, Guo H et al (2009) Prognostic significance of FTO genotype in the development of obesity in Japanese: the J-SHIPP study. Int J Obes (Lond) 33:1243-1248

39. Han X, Luo Y, Ren Q et al (2010) Implication of genetic variants near SLC30A8, HHEX, CDKAL1, CDKN2A/B, IGF2BP2, FTO, $T C F 2, K C N Q 1$, and WFS1 in type 2 diabetes in a Chinese population. BMC Med Genet 11:81

40. Sanghera DK, Ortega L, Han S et al (2008) Impact of nine common type 2 diabetes risk polymorphisms in Asian Indian Sikhs: PPARG2 (Pro12Ala), IGF2BP2, TCF7L2 and FTO variants confer a significant risk. BMC Med Genet 9:59

41. Takeuchi F, Yamamoto K, Katsuya T et al (2011) Association of genetic variants for susceptibility to obesity with type 2 diabetes in Japanese individuals. Diabetologia 54:1350-1359

42. Shu XO, Long J, Cai Q et al (2010) Identification of new genetic risk variants for type 2 diabetes. PLoS Genet 6: e1001127

43. Zhou BF, Cooperative Meta-Analysis Group of the Working Group on Obesity in China (2002) Predictive values of body mass index and waist circumference for risk factors of certain related diseases in Chinese adults: study on optimal cut-off points of body mass index and waist circumference in Chinese adults. Biomed Environ Sci 15:83-96

44. DerSimonian R, Laird N (1986) Meta-analysis in clinical trials. Control Clin Trials 7:177-188
45. Higgins JP, Thompson SG (2002) Quantifying heterogeneity in a meta-analysis. Stat Med 21:1539-1558

46. Ng MC, Tam CH, Lam VK, So WY, Ma RC, Chan JC (2007) Replication and identification of novel variants at TCF7L2 associated with type 2 diabetes in Hong Kong Chinese. J Clin Endocrinol Metab 92:3733-3737

47. Li S, Zhao JH, Luan J et al (2009) Cumulative effects and predictive value of common obesity-susceptibility variants identified by genome-wide association studies. Am J Clin Nutr 91:184-190

48. Consultation WHOE (2004) Appropriate body-mass index for Asian populations and its implications for policy and intervention strategies. Lancet 363:157-163

49. Lear SA, Humphries KH, Kohli S, Chockalingam A, Frohlich JJ, Birmingham CL (2007) Visceral adipose tissue accumulation differs according to ethnic background: results of the Multicultural Community Health Assessment Trial (M-CHAT). Am J Clin Nutr $86: 353-359$

50. Chan JC, Malik V, Jia W et al (2009) Diabetes in Asia: epidemiology, risk factors, and pathophysiology. JAMA 301:2129-2140

51. Kirkpatrick CL, Marchetti P, Purrello F et al (2010) Type 2 diabetes susceptibility gene expression in normal or diabetic sorted human alpha and beta cells: correlations with age or BMI of islet donors. PLoS One 5:e11053

52. Bravard A, Lefai E, Meugnier E et al (2011) FTO is increased in muscle during type 2 diabetes, and its overexpression in myotubes alters insulin signaling, enhances lipogenesis and ROS production, and induces mitochondrial dysfunction. Diabetes 60:258-268

53. Consortium HP-AS, Abdulla MA, Ahmed I et al (2009) Mapping human genetic diversity in Asia. Science 326:1541-1545

54. Hardy R, Wills AK, Wong A et al (2009) Life course variations in the associations between FTO and $M C 4 R$ gene variants and body size. Hum Mol Genet 19:545-552

55. Sha BY, Yang TL, Zhao LJ et al (2009) Genome-wide association study suggested copy number variation may be associated with body mass index in the Chinese population. J Hum Genet 54:199-202

56. Hu C, Zhang R, Wang $\mathrm{C}$ et al (2009) PPARG, KCNJ11, CDKAL1, $C D K N 2 A-C D K N 2 B, I D E-K I F 11-H H E X, I G F 2 B P 2$ and SLC3OA8 are associated with type 2 diabetes in a Chinese population. PLoS One 4:e7643

57. Wen J, Ronn T, Olsson A et al (2010) Investigation of type 2 diabetes risk alleles support CDKN2A/B, CDKAL1, and TCF7L2 as susceptibility genes in a Han Chinese cohort. PLoS One 5:e9153

58. Cha S, Koo I, Park BL et al (2009) Genetic effects of FTO and MC4R polymorphisms on body mass in constitutional types. Evid Based Complement Alternat Med. doi:10.1093/ecam/nep162

59. Takeuchi F, Serizawa M, Yamamoto K et al (2009) Confirmation of multiple risk loci and genetic impacts by a genome-wide association study of type 2 diabetes in the Japanese population. Diabetes 58:1690-1699 